\title{
Cluster and Cross-correlation Analysis of some Physiological Parameters by Various Desulfovibrio sp. and Desulfomicrobium sp. Bacterial Strains of the Human Intestine
}

\author{
Ivan V. Kushkevych ${ }^{1 *}$ and Yurij J. Beno ${ }^{2}$ \\ ${ }^{1}$ Faculty of Pharmacy, University of Veterinary and Pharmaceutical Sciences Brno, Palackeho 1/3, CZ-61242 Brno, Czech Republic \\ ${ }^{2}$ Faculty of Biology, Ivan Franko National University of Lviv, Hrushevsky St. 4, Lviv 79005, Ukraine
}

Received: November 25, 2013; Accepted: December 28, 2013; Published: December 30, 2013

*Corresponding author: Ivan V. Kushkevych, University of Veterinary and Pharmaceutical Sciences Brno, Palackeho 1/3, CZ-61242 Brno, Czech Republic Tel: +420-732-215-046; Fax: +420-541-240-605; E-mail: ivan.kushkevych@gmail.com

\begin{abstract}
In this paper we present a cluster analysis of the dissimilatory sulfate reduction parameters by the sulfate-reducing bacteria Desulfovibrio sp. and Desulfomicrobium sp. various strains isolated from human intestine. The studied bacterial strains accumulate hydrogen sulfide and acetate in the intestine. These substances are toxic and effect on the intestinal cells metabolism causing various diseases. Clustering of the bacterial growth, the sulfate and lactate usage as well as hydrogen sulfide and acetate accumulation by the Desulfovibrio sp. and Desulfomicrobium sp. various strains has been carried out. The studied strains have formed different clusters by the specific parameters of sulfate reduction. Correlation coefficients $(r)$ between parameters of dissimilatory sulfate reduction by the Desulfovibrio sp. and Desulfomicrobium sp. strains as well as crosscorrelation between the parameters have been determined. Inverse (negative) strong correlation between biomass and sulfate $(r=-0.975$; $r=-0.983$ for both studied Desulfovibrio sp. and Desulfomicrobium sp. strains, respectively), biomass and lactate $(r=-0.974 ; r=$ -0.958), sulfate and sulfide ( $r=-0.97 ; r=-0.958)$, sulfate and acetate $(r=-0.844 ; r=-0.92)$, lactate and acetate $(r=-0.912 ; r=-0.957)$, lactate and sulfide $(r=-0.976 ; r=-0.941)$ have been determined. Variance analysis of the bacterial strains growth has showed that proportions of the temperature and pH effect are $97.53 \%(P>0.99)$ and $97.83 \%(P>0.99)$, respectively, for Desulfovibrio sp. strains as well as $92.99 \%(P>0.99)$ and $91.32 \%(P>0.99)$, respectively, for growth of Desulfomicrobium sp. bacterial strains. Such studies might help to construct models of intensive sulfate reduction by the studied strains, and also a more detailed understanding of the role these strains have in the development of bowel diseases as well as in predicting the development of diseases in the gastrointestinal tract, by providing further details on the etiology of bowel diseases which are very important for the clinical diagnosis of these disease types.
\end{abstract}

Key words: Cluster analysis; Cross-correlation; Sulfate-reducing bacteria; Desulfovibrio; Desulfomicrobium; Inflammatory Bowel diseases

\section{Introduction}

Sulfate-reducing bacteria Desulfovibrio and Desulfomicrobium genera belong to the micro flora of the human intestine $[1,2]$. Disturbance of the ratio of these bacteria, due to other intestinal microorganisms, may lead to metabolic disorders $[3,4,5,6]$. Previous studies have shown that an increase of sulfate-reducing bacteria in the human intestine causes the active accumulation of hydrogen sulfide and acetate which are toxic to the epithelial cells of the intestine [6,7]. High concentrations of these compounds can cause carcinogenic effects and cell apoptosis $[7,8]$. It is important to obtain and isolate new strains of sulfatereducing bacteria, to study their metabolic pathways of synthesis of the toxic compounds and investigate the cluster links as well as the correlations between these strains and their physiological parameters.

Multivariate statistical analysis refers to multiple advanced techniques for examining relationships among multiple variables at the same time. This analysis includes cluster analysis, principal components method and factor analysis. The features of multivariate analysis most vividly demonstrate the classification of objects of cluster analysis and the investigation of connections that is, factor analysis.

The possibility of computer processing of large databases has made it possible to carry out cluster analysis $[9,10]$. The cluster analysis involves the selection of compact, widely separated groups of objects. This analysis finds a "natural" partition set to the region of cluster objects. It is used when the source data is presented in a matrix of closeness or remoteness between the objects or when the source data is presented as points in a multidimensional space. The most common data of the second type can be analyzed through a cluster analysis, which focuses on the allocation of remote groups within which the objects are similar. A selected distance between objects is the nodal point of 
the study, which preconditions the final partition of the objects into classes through an algorithm partitioning [10,11]. There are lots of algorithms in a cluster analysis. Based on the type of a cluster construction the algorithms can be divided into the standard and non-standard ones [12].

The processes of biosystems autoregulation, their survival and development are largely dependent on the fluctuations of parameters. These processes are the time-spatial organization of bacterial growth $[13,14]$. During the simultaneous separation of bacterial cells, accompanied by the fluctuations of biophysical and metabolic parameters, the subordination of the biological clock rhythm serves as a determining factor in the regulation of oscillatory dynamics of these processes [14]. In previous studies ten various Desulfovibrio sp. strains and ten various Desulfomicrobium sp. strains were isolated from the human intestine and identified. The growth of these bacteria, their use of sulfate and lactate as well as the production of hydrogen sulfide and acetate were also studied [12].

The aim of this research was to study the cluster relationship between the changes of certain physiological parameters of various Desulfovibrio sp. and Desulfomicrobium sp. bacterial strains of the human intestine, and to carry out the crosscorrelation as well as two-factor analysis of these parameters.

\section{Material and Methods}

The material for analysis is the experimental data of changes in time of the sulfate reduction parameters (bacterial growth, the sulfate and lactate usage and sulfide and acetate formation during ten days of cultivation) by the Desulfovibrio sp. (SRB Vib-1, SRB Vib-2, SRB Vib-3, SRB Vib-4, SRB Vib-5, SRB Vib-6, SRB Vib7, SRB Vib-8, SRB Vib-9, SRB Vib-10) and Desulfomicrobium sp. (SRB Rod-1, SRB Rod-2, SRB Rod-3, SRB Rod-4, SRB Rod-5, SRB Rod-6, SRB Rod-7, SRB Rod-8, SRB Rod-9, SRB Rod-10) bacterial strains isolated from the human intestine [15].

Using the experimental data, the basic statistical parameters ( $\mathrm{M}$ - mean, $\mathrm{m}$ - standard error, $\mathrm{M} \pm \mathrm{m}$ ) have been calculated. For the estimation of the reliability between the statistical characteristics, Student's $t$-test was used [16]. The difference was reliable when $P>0.95$ [17]. Statistical processing of the results has been performed using packet Excel, Origin [18] and Statistica programmers.

Comparative analysis of parameters of sulfate reduction by the various Desulfovibrio sp. and Desulfomicrobium sp. bacterial strains, differentiation of the strains and assessment of their level of similarity were performed using the method of cluster analysis. Cluster analysis is a method of grouping also multidimensional objects which are based on the representation of the results of the individual observations to suitable space, followed by separation of the groups as «clusters» (or taxons) $[9,12]$.

The study of temporal correlations between the growth of bacteria and their metabolic processes has been carried out using the correlation analysis in time. This method makes possible to identify not only the relationship between the parameters but it also raises questions about the possibility of their relationship.
The values of the defined functional parameters, namely the bacterial growth, the sulfate and lactate usage as well as the formation of hydrogen sulfide and acetate by the Desulfovibrio sp. and Desulfomicrobium sp. strains were used in the correlations study.

The shear sign that corresponds to the accurate correlation coefficient indicates which of the rows have the ability to anticipate the outcome of other rows. The offset values in the time ahead reflect the cause of the relationships. It is difficult to determine the nature of a relationship when it is negative. A positive correlation coefficient value may determine different landslides of some processes. Such two-directional connections can be interpreted in two ways: a real cross-correlation between the rows or by correlations caused due to the inherent fluctuations of the period $[19,20]$.

The cross-correlation analysis is obtained from the correlograms for Desulfovibrio sp. and Desulfomicrobium sp. strains. A correlogram is a sequence of values of the correlation coefficients determined by the convergence of different shifts of a series on the other. Pitch shift, the time interval between two adjacent measurements, equals 24 hours. The reliability of the results is determined by the table limit values of the correlation coefficients, which guarantees the specified level of significance, depending on the amount of aggregate (in our case, $\mathrm{p}<0.05$ ) [13].

\section{Results}

The cluster analysis of the parameters of the bacterial growth, the sulfate and lactate usage, and hydrogen sulfide and acetate production by the studied strains was performed (figure 1). As a result of the clustering of the growth parameters, five independent clusters are formed. A dendrogram shows the greatest similarity between Desulfovibrio sp. strain Vib-10 with Desulfomicrobium sp. strain Rod-10 and Desulfomicrobium sp. strain Rod-8 with Desulfomicrobium sp. strain Rod-7 by the tested parameter. The strains Desulfovibrio sp. Vib-9 with Desulfovibrio sp. Vib-7 and Desulfovibrio sp. Vib-4 as well as Desulfovibrio sp. Vib-8 and Desulfovibrio sp. Vib-6 have formed an isolated cluster. The same cluster was formed by the bacterial strains Desulfomicrobium sp. Rod-9 with Desulfomicrobium sp. Rod-4 and Desulfomicrobium sp. Rod-6 as well as Desulfovibrio sp. Vib5 with Desulfomicrobium sp. Rod-3. The strains Desulfovibrio sp. Vib-3 and Desulfovibrio sp. Vib-1 and Desulfovibrio sp. Vib-2 were the most varied by parameter tested comparisons with strains Desulfomicrobium sp. Rod-5 and Desulfomicrobium sp. Rod-2 and Desulfomicrobium sp. Rod-1, forming two separate isolated clusters (figure $1 \mathrm{~A}$ ).

The parameters of intensity of sulfate utilization by the studied strains are most similar to each other, allowing them to combine into clusters. In this case there is also the formation of five clusters, consisting of strains Desulfovibrio sp. Vib-7 with Desulfovibrio sp. Vib-4 and Desulfovibrio sp. Vib-8 in one cluster and the strains Desulfovibrio sp. Vib-9 with Desulfomicrobium sp. Rod-8 and Desulfomicrobium sp. Rod-6 in another cluster. In this series of experiments the strains Desulfovibrio sp. Vib6 with Desulfovibrio sp. Vib-3, Desulfovibrio sp. Vib-5 and 

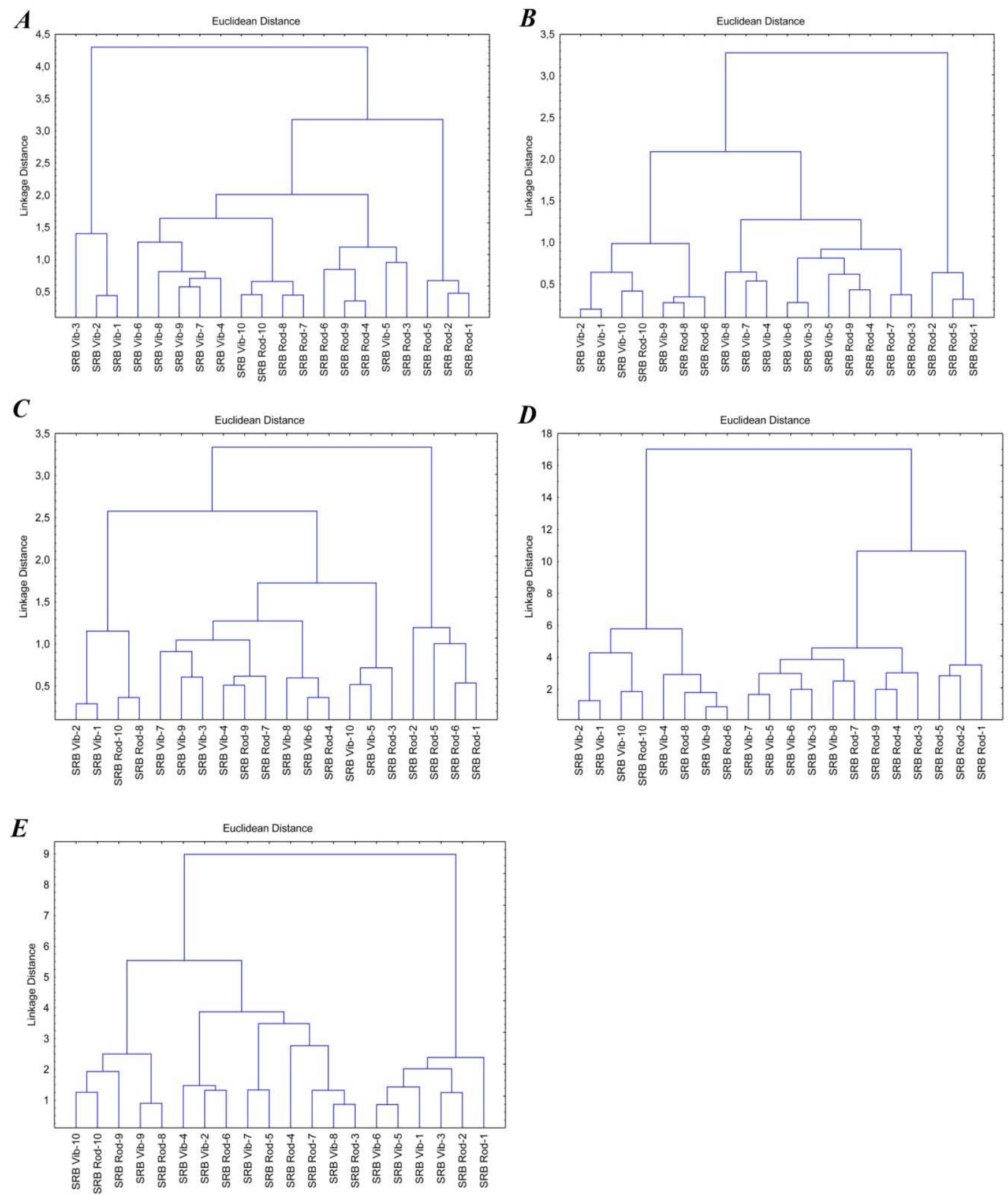

Figure 1: Dendrograms for the various Desulfovibrio sp. and Desulfomicrobium sp. strains showing cluster analysis of the parameters: A - the growth; $\mathrm{B}$ - the sulfate usage; C - the hydrogen sulfide production; D - the lactate usage; $\mathrm{E}$ - the acetate production (Complete Linkage). 
Desulfomicrobium sp. Rod-9 with Desulfomicrobium sp. Rod-4 as well as Desulfomicrobium sp. Rod-7 with Desulfomicrobium sp. Rod-3 formed an isolated cluster. However, the strains Desulfovibrio sp. Vib-10 with Desulfomicrobium sp. Rod-10 were more similar to the strains Desulfovibrio sp. Vib-1 with Desulfovibrio sp. Vib-2, which was not observed in the previous series of experiments. Moreover, the Desulfomicrobium sp. Rod-5 with Desulfomicrobium sp. Rod-1 and Desulfomicrobium sp. Rod2 formed a stable isolated cluster (Figure 1B).

The resulted clustering of quantitative formation of hydrogen sulfide has showed that strains Desulfovibrio sp. Vib9 with Desulfovibrio sp. Vib-3 and Desulfovibrio sp. Vib-7 were combined in the cluster with strains Desulfomicrobium sp. Rod7 and Desulfovibrio sp. Vib-4 with Desulfomicrobium sp. Rod-9. Moreover, there are two formed clusters by bacterial strains Desulfovibrio sp. Vib-6 with Desulfomicrobium sp. Rod-4 and Desulfovibrio sp. Vib-8 as well as Desulfovibrio sp. Vib-10 with Desulfovibrio sp. Vib-5 and Desulfomicrobium sp. Rod-3. In this series of experiments the strains Desulfovibrio sp. Vib-2 with Desulfovibrio sp. Vib-1 and Desulfomicrobium sp. Rod-10 with Desulfomicrobium sp. Rod-8 as well as Desulfomicrobium sp. Rod-1 with Desulfomicrobium sp. Rod-6 and Desulfomicrobium sp. Rod-5 and Desulfomicrobium sp. Rod-2 have formed separate isolated independent clusters (figure 1C).

The clustering of the parameters of lactate usage by the studied strains has showed that the strains Desulfovibrio sp. Vib-7 with Desulfovibrio sp. Vib-5 and Desulfovibrio sp. Vib6 with Desulfovibrio sp. Vib-3 as well as Desulfovibrio sp. Vib-8 with Desulfomicrobium sp. Rod-7 have formed in the clusters. Moreover, the bacterial strains Desulfomicrobium sp. Rod-9 with Desulfomicrobium sp. Rod-4 and Desulfomicrobium sp. Rod-3 as well as Desulfomicrobium sp. Rod-5 with Desulfomicrobium sp. Rod-2 and Desulfomicrobium sp. Rod-1 have formed two clusters. There is also the formation of other clusters by bacterial strains Desulfovibrio sp. Vib-9 with Desulfomicrobium sp. Rod-6, Desulfomicrobium sp. Rod-8 and Desulfovibrio sp. Vib-4 as well as Desulfovibrio sp. Vib-10 with Desulfomicro-bium sp. Rod-10 and Desulfovibrio sp. Vib-1 with Desulfovibrio sp. Vib-2. In this case, there is a clear division formed resulting in two isolated clusters on the dendrogram (figure 1D).

The results of the cluster analysis of the parameters of the acetate production by the strains have showed the separate formation of clusters on the dendrogram (figure 1E). Bacterial strains Desulfovibrio sp. Vib-2 with Desulfomicrobium sp. Rod-6, Desulfovibrio sp. Vib-4, Desulfovibrio sp. Vib-7 with Desulfomicrobium sp. Rod-5 as well as Desulfovibrio sp. Vib-8 with Desulfomicrobium sp. Rod-3, Desulfomicrobium sp. Rod-7, Desulfomicrobium sp. Rod-4 were combined in a clear division into three isolated clusters. The strains Desulfovibrio sp. Vib6 with Desulfovibrio sp. Vib-5 and Desulfovibrio sp. Vib-1 as well as Desulfovibrio sp. Vib-3 with Desulfomicrobium sp. Rod2 and Desulfomicrobium sp. Rod-1 were the most varied by parameter testing, compared to strains Desulfovibrio sp. Vib-9 with Desulfomicrobium sp. Rod-8, Desulfovibrio sp. Vib-10 with Desulfomicrobium sp. Rod-10 and Desulfomicrobium sp. Rod-
9, which have formed two separate isolated clusters on the dendrogram.

Thus the cluster analysis on the basis of the different parameters of the bacterial growth, the sulfate and lactate usage, and hydrogen sulfide and acetate production by the studied various Desulfovibrio sp. and Desulfomicrobium sp. bacterial strains has been carried out. Cluster analysis involves the selection of compact, far-flung groups of objects which are found "natural" partitioning aggregate area cluster objects. It applies when original data is represented as matrices of proximity or distance between objects or as points in a multidimensional space.

Cross-correlation analysis is the tool most commonly used in the analysis of multiple time series. Its application to biological objects merits special attention. The next stage of our research was to carry out a cross-correlation analysis between the sulfate reduction parameters by the various Desulfovibrio sp. and Desulfomicrobium sp. strains.

The results of the cross-correlation analysis showed a strong correlation in pairs of the following parameters: biomass and sulfate, biomass and lactate, sulfate and sulfide, sulfate and acetate, lactate and acetate, lactate and sulfide, biomass and sulfide, biomass and acetate, lactate and sulfate, acetate and sulfide. The processes that describe the changes in these parameters closely conjugate in the various Desulfovibrio sp. and Desulfomicrobium sp. strains (figure 2, 3). The cross- correlograms of the studied parameters are similar for both Desulfovibrio $\mathrm{sp}$. and Desulfomicrobium sp. strains.

The correlation coefficients $(r)$ between the parameters of dissimilatory sulfate reduction were defined (table 1). Inverse (negative) strong correlation between biomass and sulfate, biomass and lactate, sulfate and sulfide, sulfate and acetate, lactate and acetate, lactate and sul-fide have been determined for all studied Desulfovibrio sp. and Desulfomicrobium sp. strains. However, between parameters of the biomass and sulfide, biomass and acetate, lactate and sulfate, acetate and sulfide, a direct (positive) strong correlation has been observed. The correlation coefficients by different time shifts vary, however they are closely related by the value and sign. Perhaps, the mechanisms and rates of sulfate reduction by these studied bacterial strains are identical and connected. A small difference between crosscorrelograms may be due to a change in the phase relationship between them at the beginning and ending of observation. The time shifts of certain rows in different directions are showing reliable cross-correlation relationships. It shows the stability of the relationship of these parameters by the periodic components. The correlation arises during the shift in the forward and reverse direction. If the correlation value is quite low, it may be due to the nonlinear nature of change indicators, instability period or the presence of other periodic components.

Based on a coherent picture of temporal dependencies between processes, which determine the changes of the sulfate reduction parameters by the Desulfovibrio sp. and Desulfomicrobium sp. strains as well as intensity of their energy 

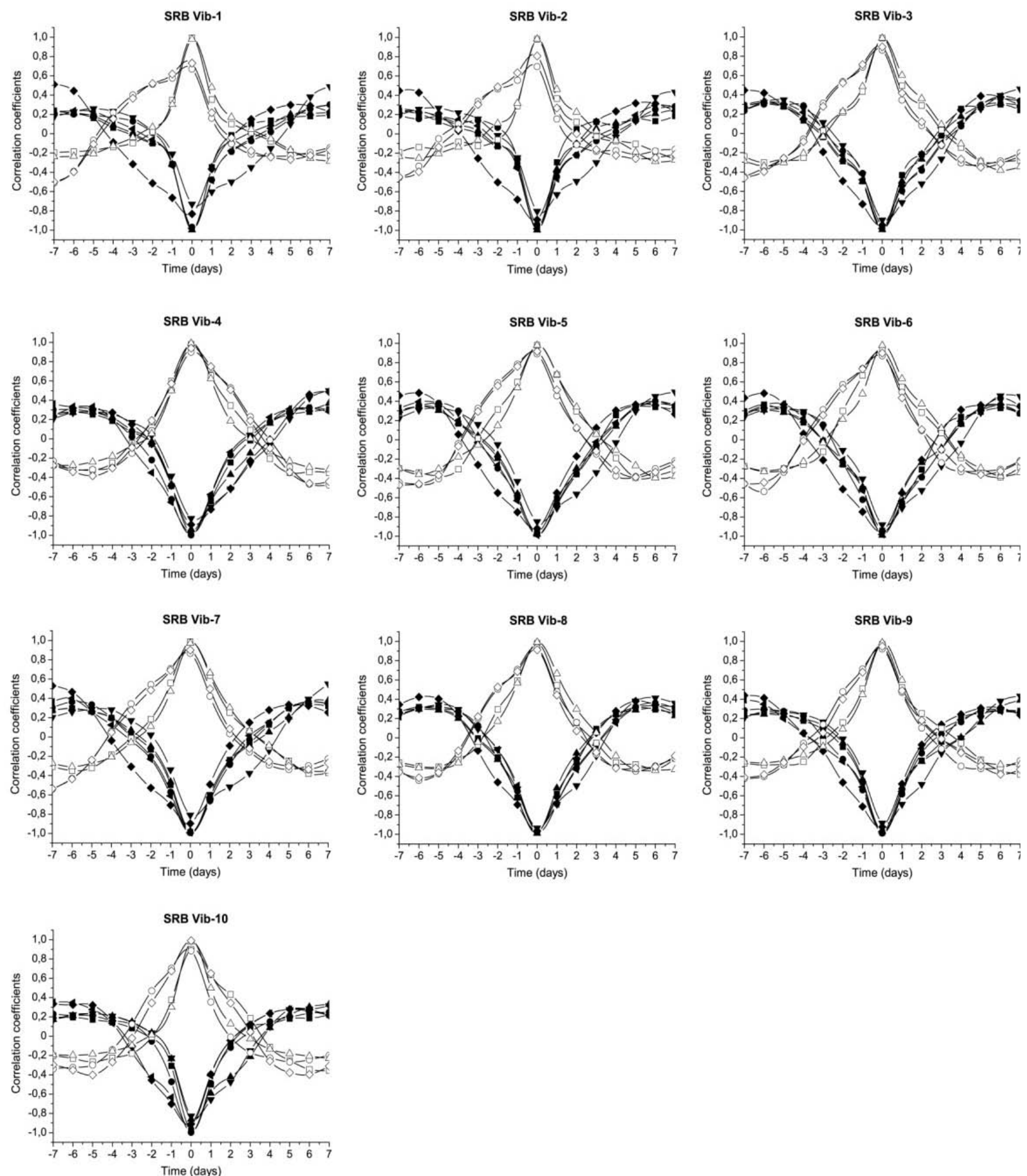

Figure 2: Cross-correlation between dissimilatory sulfate reduction parameters by the various Desulfovibrio sp. strains: -匹- biomass and sulfate; - - biomass and lactate; - $\mathbf{\Delta}$ - sulfate and sulfide; - $\mathbf{\nabla}$ - sulfate and acetate; - - lactate and acetate; - $\mathbf{-}$ - lactate and sulfide; $-\square$ - biomass and sulfide; - - - biomass and acetate; $-\Delta$ - lactate and sulfate; $-\diamond$ - acetate and sulfide.

Citation: Kushkevych IV, Beno YJ (2013) Cluster and cross-correlation analysis of some physiological parameters by various Desulfovibrio sp. and Desulfomicrobium sp. bacterial strains of the human intestine. SOJ Microbiol Infect Dis 1(1): 9. DOI: http:// 

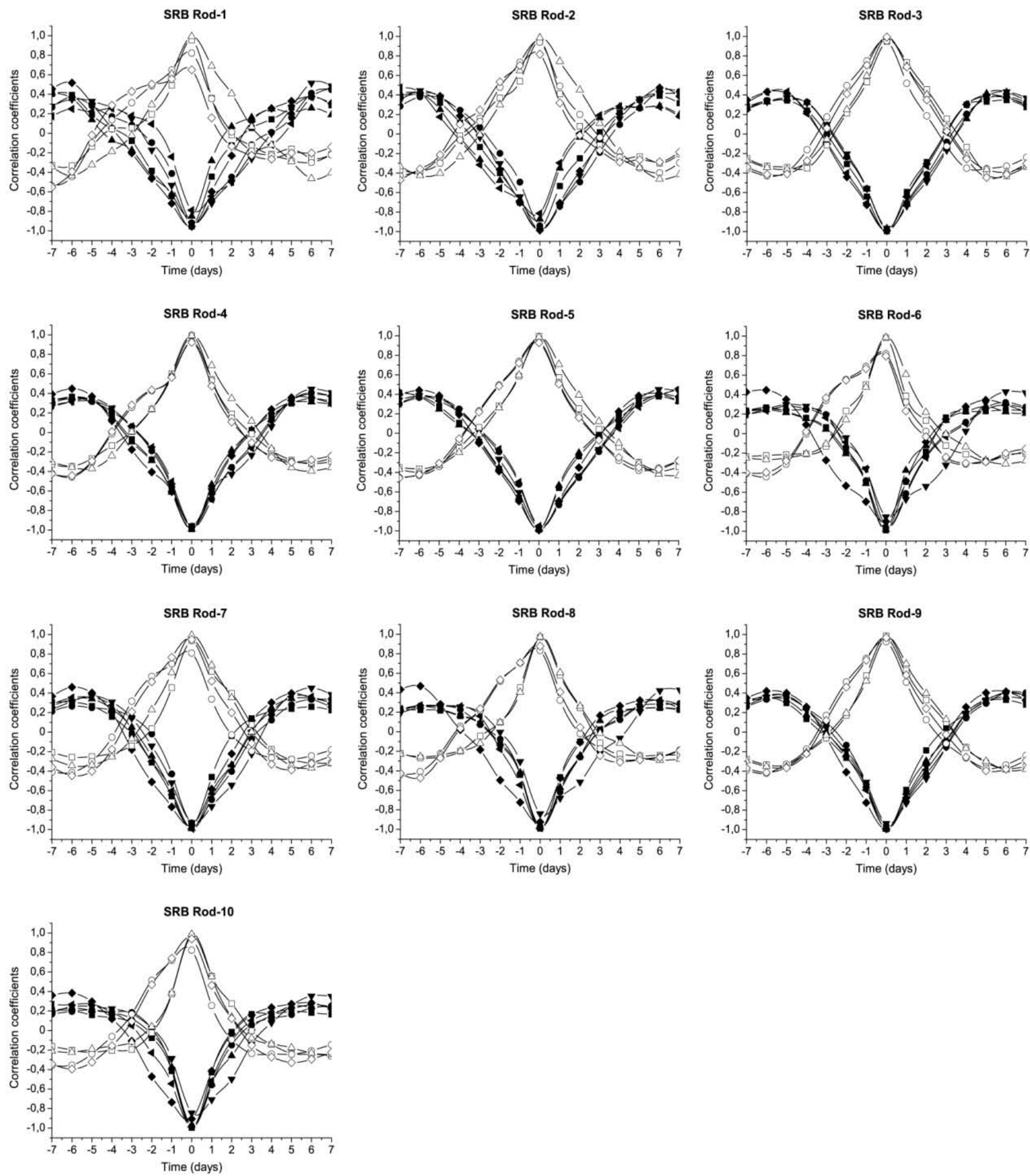

Figure 3: Cross-correlation between dissimilatory sulfate reduction parameters by the various Desulfomicrobium sp. strains: - $\mathbf{- 1}$ biomass and sulfate; - $\bullet$ - biomass and lactate; $-\boldsymbol{\Lambda}$ - sulfate and sulfide; $-\boldsymbol{\nabla}$ - sulfate and acetate; $-\bullet$ - lactate and acetate; - $\mathbf{-}$ - lactate and sulfide; $-\square-$ biomass and sulfide; $-\odot-$ biomass and acetate; $-\Delta$ - lactate and sulfate; $-\diamond$ - acetate and sulfide. 
metabolism, we can assume that these strains have the same clock mechanism regulating metabolism.

Thus the performed analysis of joint dynamics of metabolic processes enables a deeper understanding of their temporal organization at different stages of sulfate reduction. Identified analogy in time between the dynamics of energy metabolism and sulfate reduction processes confirmed the versatility of the relationships.

A two-factor analysis of variance is used when you have two independent variables (or factors) and want to examine the effect of each of those variables independently as well as when in interaction with each other on a dependent variable [14]. Quantitative evaluation of the influence of growth regulators by methods of variance analysis was carried out. To exclude the influence of natural increase of the bacterial number in the process of their growth (caused by the time factor), all incentive effects were assessed by the regression line. From the experimental material, two-factor analysis was performed on the temperature and $\mathrm{pH}$ effects which can be regulators of bacterial growth (Tables 2 and 3). This analysis enabled evaluation and comparison of the relative proportion of their influence, individual effect of either $\mathrm{pH}$ or temperature and also the influence of other uncontrollable factors in experiments on the studied parameter of bacterial growth. Variance analysis of Desulfovibrio sp. bacterial strains growth has showed that the effect of temperature and $\mathrm{pH}$ as a proportion is $97.53 \%(P>0.99)$ and $97.83 \% \quad(P>0.99)$, respectively. Moreover, unaccounted factors are $2.47 \%$ and $2.17 \%$ respectively for temperature and pH effect (table 2).

The proportion of the temperature and $\mathrm{pH}$ effect is $92.99 \%$ $(P>0.99)$ and $91.32 \% \quad(P>0.99)$, respectively, for variance analysis of the growth of Desulfomicrobium sp. bacterial strains. Unaccounted factors for temperature and $\mathrm{pH}$ effect are $7.01 \%$ and $8.68 \%$, respectively (table 3 ).

This performed variation analysis will help to establish the variability of studied growth parameter, which is not associated with changes in its level caused by the presence or absence of regulatory experimental influences. The relative proportion of the variability was caused by the action of unaccounted factors.

\section{Discussion}

The Desulfovibrio and Desullfomicrobium genera belong to anaerobic, gram-negative, sulfate-reducing bacteria (SRB)
$[1,5]$. They use different nutrient substances that a human consumes [5]. SRB use sulfate ions, from the substances, as electron acceptors and organic compounds (lactate, acetate, $\mathrm{H}_{2}$, fatty acids, ethanol, dicarboxylic acids and other) as electron donors. Some SRB species can grow autotrophically using $\mathrm{H}_{2}, \mathrm{CO}_{2}$ and sulfate. The bacteria can use ammonium salts as nitrogen sources as well as assimilating molecular nitrogen [1]. It has been suggested that some SRB species might be responsible for the development of some forms of colon cancer, given the fact that these microorganisms produce hydrogen sulfide and acetate affecting the metabolism of intestinal cells causing ulcerative enterocolitis $[3,5]$. These bacteria have been isolated from pyogenic liver abscesses and they have been found to cause septicaemia, bacteremia and periodontal disease [6,21]. It is thought that SRB combined with other infections can cause a variety of diseases including cholecystitis, brain abscesses and abdominal cavities $[1,5,6]$. The species of the Desulfovibrio genus are often isolated among all SRB during illness. These bacteria have the most pathogenic role of all SRB species $[3,2,6]$ Bacteria of the Desulfovibrio genus are isolated during both mono- and polymicrobial infections of the gastrointestinal tract [1,2].

The results of cluster analysis of tested parameters have shown that studied bacteria form various clusters. The similarity between various Desulfovibrio sp. and Desulfomicrobium sp. strains has been established. Similar clusters were obtained by Dzierzewicz Z et al.[22], for fifteen (soil and intestinal) strains of Desulfovibrio desulfuricans species that were typed by PCR with the use of primers specific for repetitive extragenic palindromic and enterobacterial repetitive intergenic consensus sequences. Distinct fingerprint patterns of two isolates derived from the same patient pointed to the different origin of both strains. There is also cluster analysis showing the presence of three distinct clusters among $D$. desulfuricans for all intestinal strains (group 1) which were approximately identical (98.2 to $99.8 \%$ similarity). Similarity was evaluated in a group 2 , containing six soil strains (DV-1/84, DV-2/84, DV-4/84, DV-5/84, DV-6/84 and DV-8/84). The members of this group had $87 \%$ similarity with the soil strain. All intestinal strains and soil strains were similar at the 85.5\% level. Strains DV-3/84 DV-7/84 (group3) showed 76.6\% similarity to each other and they were similar to all other strains at the $67.6 \%$ level [22].

A solution to the problem of cluster analysis is separation that provides some optimal condition. These criteria may be a function expressing the desirability of different levels of partitions and

Table 1: Correlation coefficients $(r)$ between parameters of dissimilatory sulfate reduction by the Desulfovibrio sp. and Desulfomicrobium sp. bacterial strains.

\begin{tabular}{|c|c|c|c|c|c|c|c|c|c|c|}
\hline \multicolumn{6}{|c|}{ Desulfovibrio sp. Vib-7 } & \multicolumn{5}{|c|}{ Desulfomicrobium sp. Rod-9 } \\
\hline & Biomass & Sulfate & Sulfide & Lactate & Acetate & Biomass & Sulfate & Sulfide & Lactate & Acetate \\
\hline Biomass & 1 & -0.975 & 0.969 & -0.974 & 0.85 & 1 & -0.983 & 0.9685 & -0.958 & 0.881 \\
\hline Sulfate & -0.975 & 1 & -0.97 & 0.982 & -0.844 & -0.983 & 1 & -0.958 & 0.9853 & -0.92 \\
\hline Sulfide & 0.969 & -0.97 & 1 & -0.976 & 0.894 & 0.9685 & -0.958 & 1 & -0.941 & 0.8845 \\
\hline Lactate & -0.974 & 0.982 & -0.976 & 1 & -0.912 & -0.958 & 0.9853 & -0.941 & 1 & -0.957 \\
\hline Acetate & 0.85 & -0.844 & 0.894 & -0.912 & 1 & 0.881 & -0.92 & 0.8845 & -0.957 & 1 \\
\hline
\end{tabular}


Table 2: Two-factor analysis of variation, calculating the proportion of the investigated factors influencing Desulfovibrio sp. Vib-7 growth on sixth day.

\begin{tabular}{|c|c|c|c|c|c|}
\hline Factor of influence & $\begin{array}{c}\text { Sum of squares } \\
\text { SS }\end{array}$ & $\begin{array}{c}\text { Proportion of } \\
\text { influence } \\
\eta^{2}, \%\end{array}$ & $\begin{array}{c}\begin{array}{c}\text { Fisher } \\
\text { coefficient }\end{array} \\
F \\
\text { (practical) }\end{array}$ & $\begin{array}{c}\text { Fisher coefficient } \\
F \\
\text { (critical) }\end{array}$ & $\begin{array}{c}\text { Reliability } \\
P\end{array}$ \\
\hline Proportion of temperature effect & 62.73 & 97.53 & \multirow{3}{*}{444.23} & \multirow{3}{*}{2.59} & \multirow{3}{*}{0.999} \\
\hline Unaccounted factors & 1.59 & 2.47 & & & \\
\hline Total & 64.32 & 100 & & & \\
\hline Proportion of pH effect & 52.37 & 97.83 & \multirow{3}{*}{473.72} & \multirow{3}{*}{2.25} & \multirow{3}{*}{0.999} \\
\hline Unaccounted factors & 1.16 & 2.17 & & & \\
\hline Total & 53.53 & 100 & & & \\
\hline
\end{tabular}

Table 3: Two-factor analysis of variation, calculating the proportion of the investigated factors influencing Desulfomicrobium sp. Rod-9 growth on sixth day.

\begin{tabular}{|c|c|c|c|c|c|}
\hline Factor of influence & $\begin{array}{c}\text { Sum of squares } \\
\text { SS }\end{array}$ & $\begin{array}{c}\text { Proportion of } \\
\text { influence } \\
\eta^{2}, \%\end{array}$ & $\begin{array}{c}\text { Fisher } \\
\text { coefficient } \\
F \\
\text { (practical) }\end{array}$ & $\begin{array}{c}\text { Fisher coefficient } \\
F \\
\text { (critical) }\end{array}$ & $\begin{array}{c}\text { Reliability } \\
P\end{array}$ \\
\hline Proportion of temperature effect & 43.34 & 92.99 & \multirow{3}{*}{149.31} & \multirow{3}{*}{2.58} & \multirow{3}{*}{0.999} \\
\hline Unaccounted factors & 3.26 & 7.01 & & & \\
\hline Total & 46.61 & 100 & & & \\
\hline Proportion of pH effect & 46.73 & 91.32 & \multirow{3}{*}{110.45} & \multirow{3}{*}{2.25} & \multirow{3}{*}{0.999} \\
\hline Unaccounted factors & 4.44 & 8.68 & & & \\
\hline Total & 51.18 & 100 & & & \\
\hline
\end{tabular}

groups. This functionality is often called the objective function. The task of cluster analysis is an optimization problem, namely finding the minimum of the objective function for some given set of constraints. An example of the target function may be the sum of squares of deviations of all the intra-cluster [9].

Dzierzewicz et al. [22] have also shown that the soil bacterial group as well as the intestinal bacterial group formed two different clusters. The soil strains showed greater variability than the intestinal isolates. Based on the AP-PCR fingerprints D. desulfuricans strains were differentiated depending on their origin [22].

The correlation coefficient ranges from -1.0 to +1.0 . The closer $r$ is to +1 or -1 , the more closely the two variables are related. If $r$ is close to 0 , it means there is no linear relationship between the variables. If $r$ is positive, it means that as one variable gets larger so does the other. If $r$ is negative it means that as one gets larger, the other gets smaller (often called an «inverse» correlation). While correlation coefficients are normally reported as $r=$ (a value between -1 and +1 ), squaring the values makes them easier to understand. Values between 0.7 and $1.0(-0.7$ and -1.0$)$ indicate a strong positive (negative) linear relationship via a firm linear rule [19].

Cross-correlation analysis is basically a generalization of standard linear correlation analysis. A measure of the strength of the correlation is given by the correlation coefficient. This analysis provides a correlation between two time series or two waveforms. The observations of one series are correlated with the observations of another series at various lags and leads [20]. There are three possible outcomes in the Pearson's pro-duct moment correlation $r$; positive correlation $(r=+1)$ where, as one variable rises the other variable is predic-ted to rise at a similar rate, zero $(r=0)$ or no correlation what-so-ever, or negative correlation $(r=-1)$ where, as one variable rises the other falls [19].

The cross-correlation test of two time-series data sets involves many calculations of the coefficient $r$ by time- shifting the one data set relative to the other data set. Each shift is called a «lag» and the lag time is simply the sampling period of the two time-series data sets [16, 23]. A typical cross-correlation shows enough lags in both negative and positive directions to show the cyclical relationship of the two sets of data. In crosscorrelation analysis, waveforms that alternate are out-of-phase from each other and will have a negative relationship, whereas waveforms that are synchronous will be in-phase and have a positive relationship [20]. The strength of the relationship between two waveforms will be perfect at \pm 1 and will diminish to a minimum when approaching 0 . A high degree of symmetry or stability along the $\mathrm{X}$ axis indicates a stable relationship between the two waveforms. However, as the relationship between waveforms varies, decreasing correlation values beyond zero lag are established, indicating a lack of stability in the relationship $[20,23]$.

Our results, shown by variance analysis method, indicate that a proportion of temperature and $\mathrm{pH}$ has an effect on bacterial growth. This analysis is based on testing for the significance of the magnitude of effect of two or more treatments, taking into account the variance within and between treatment classes. It is a highly flexible analytical approach that allows investigators to simultaneously assess the contributions of multiple factors to bacterial growth, including effects such factors as medium composition, temperature, $\mathrm{pH}$, bacteria age, time of their growth, as well as interactions between factors $[14,16]$. 
In summary, we conclude that the method of cluster analysis has helped establish similarity between Desulfovibrio sp. and Desulfomicrobium sp. various bacterial strains by testing different parameters of the dissimilatory sulfate reduction. Cross-correlations of their physiological parameters has helped identify variables which are leading indicators of other variables, and also how much one variable is predicted to change in relation the other. The proportion of temperature and $\mathrm{pH}$ effects on bacterial growth by the variance analysis has been demonstrated. The cluster analysis data can help to select some strains by the specific parameters for conducting future studies, and also more detailed understanding of etiology role of Desulfovibrio sp. and Desulfomicrobium sp. bacterial strains in the development of the diseases.

\section{Conclusions}

The cluster analysis of different sulfate reduction parameters by various Desulfovibrio sp. and Desulfomicrobium sp. bacterial strains showed that these strains were closely related by one parameter while still being separated by other parameters of sulfate reduction. The studied bacterial strains, which are closely related by specific parameters, can be selected for future studies.

Cross-correlations of dissimilatory sulfate reduction parameters can be selected to construct models of intensive sulfate reduction by the studied Desulfovibrio sp. and Desulfomicrobium sp. bacterial strains, and also a more detailed understanding of the role these strains have in the development of bowel diseases.

Two-factor analysis, calculating the proportion of the investigated factors which influence growth of the $D$. desulfuricans and Desulfomicrobium sp. strains, on the sixth day, has been demonstrated. This analysis is a valuable way of studying the proportion of temperature and $\mathrm{pH}$ effects on bacterial growth of studied strains.

\section{Acknowledgements}

The authors express their gratitude to Dr. Roman Fafula, $\mathrm{PhD}$, Assistant Professor at the Biophysics Department at the Danylo Halytsky Lviv National Medical University for his help in conducting the statistical analysis. In addition to, Lucy Mclennan and Robyn Cochrane from Forensic Biology, Immunology and Microbiology at University of Strathclyde (Scotland, United Kingdom) for their assistance and critical reading of the manuscript.

\section{References}

1. Barton LL, Hamilton WA (2007) Sulphate-reducing bacteria. Environmental and Engineered Systems. Cambridge University Press, p. 553.

2. Gibson GR, Cummings JH, Macfarlane GT (1991) Growth and activities of sulphate-reducing bacteria in gut contents of health subjects and patients with ulcerative colitis. FEMS Microbiology Letters 86(2): 103-111.
3. Cummings JH, Macfarlane GT, Macfarlane S (2003) Intestinal bacteria and ulcerative colitis. Curr Issues Intest Microbiol 4(1): 9-20.

4. Gibson GR, Macfarlane S, Macfarlane GT (1993) Metabolic interactions involving sulphate-reducing and methanogenic bacteria in the human large intestine. FEMS Microbiol Ecol 12(2): 117-125.

5. Kushkevych IV, Moroz OM (2012) Growth of various strains of sulfatereducing bacteria of human large intestine. Sci Int J Biological studies/ Studia Biologica 6(3): 115-124.

6. Loubinoux J, Mory F, Pereira IA, Le Faou AE (2000) Bacteremia caused by a strain of Desulfovibrio related to the provisionally named Desulfovibrio fairfieldensis. J Clin Microbiol 38(2): 931-934.

7. Kushkevych IV (2012) Sulfate-reducing bacteria of the human intestine. II. The role in the diseases development. Sci Int J Biol Studies/Studia Biologica 6(2): 221-250.

8. Pitcher MC, Cummings JH (1996). Hydrogen sulphide: a bacterial toxin in ulcerative colitis? Gut 39(1): 1-4.

9. Djuran B, Odell P (1977) Cluster analysis. Moscow: Statistics, p128.

10. Dronov SB (2003) Multivariate statistical analysis. Barnaul: Publishing House of Altai State University Press, p. 213.

11.Jambju M (1988) Hierarchical cluster analysis and compliance. Statistics, Moscow, p. 342.

12. Mandel ID (1988) Cluster analysis. Statistics. Moscow, p. 176.

13. Derkach MP, Humetsky RJ, Chaban ME (1977) A course of the variation statistics. High School, Kyiv, p. 208.

14. Lubischev AA (1986) Variance analysis in biology. Publishing House of the Moscow University, Moscow, p. 199.

15. Kushkevych IV (2013) Identification of sulfate-reducing bacteria strains of human large intestine. Sci Int J Biological studies/Studia Biologica 7: 115-132.

16. Bailey NTJ (1995) Statistical Methods in Biology. Cambridge University Press. 3rd edition. p. 252

17. Lakin GF (1990) Biometry. High School, Moscow, p. 352.

18. Isakova OP, Tarasevych Yu Yu, Yuzjuk YuI (2009) Processing and visualization of data with the physical experiments by usage Origin package. Book House, «LIBKOM», Moscow, p.136.

19. Chen PY, Popovich PM (2002) Correlation: Parametric and Nonparametric Measures. Sage University Papers Series on Quantitative Applications in the Social Sciences, p.104.

20. Brynjarsdottir J, Lund SH, Magnusson KG, Nielsen OK (2003) Analysis of time series for rock ptarmigan and gyrfalcon populations.

21. Langendijk PS, Kulik EM, Sandmeier H, Meyer J, van der Hoeven JS (2001) Isolation of Desulfomicrobium orale sp. nov. and Desulfovibrio strain NY682, oral sulfate-reducing bacteria involved in human periodontal disease. Int J Syst Evol Microbiol 51(3): 1035-1044.

22. Dzierzewicz Z, Szczerba J, Weglarz L, Swiatkowska L, Jasinska D, el al. (2003) Intraspecies variability of Desulfovibrio desulfuricans strains determined by the genetic profiles. FEMS Microbiol Lett 219(1): 6974.

23. Bourke P (1996) Cross Correlation. 